\title{
Article \\ Electrochemical and Fluorescent Properties of Crown Ether Functionalized Graphene Quantum Dots for Potassium and Sodium Ions Detection
}

\author{
Daniela Iannazzo ${ }^{1, *(\mathbb{D})}$, Claudia Espro ${ }^{1, * \mathbb{D}}$, Angelo Ferlazzo ${ }^{1}$, Consuelo Celesti ${ }^{1} \mathbb{D}$, Caterina Branca ${ }^{2} \mathbb{D}$ \\ and Giovanni Neri ${ }^{1}$ (D)
}

1 Department of Engineering, University of Messina, Contrada Di Dio, I-98166 Messina, Italy; angelo.ferlazzo@unime.it (A.F.); ccelesti@unime.it (C.C.); gneri@unime.it (G.N.)

2 Department of Mathematical and Computer Sciences, Physical Sciences and Earth Sciences, University of Messina, I-98166 Messina, Italy; cbranca@unime.it

* Correspondence: diannazzo@unime.it (D.I.); espro@unime.it (C.E.); Tel.: +39-0906765569 (D.I.)

check for

updates

Citation: Iannazzo, D.; Espro, C.; Ferlazzo, A.; Celesti, C.; Branca, C.; Neri, G. Electrochemical and Fluorescent Properties of Crown Ether Functionalized Graphene Quantum Dots for Potassium and Sodium Ions Detection. Nanomaterials 2021, 11, 2897. https://doi.org/ 10.3390/nano11112897

Academic Editor: Antonios Kelarakis

Received: 9 October 2021

Accepted: 27 October 2021

Published: 29 October 2021

Publisher's Note: MDPI stays neutral with regard to jurisdictional claims in published maps and institutional affiliations.

\begin{abstract}
The concentration of sodium and potassium ions in biological fluids, such as blood, urine and sweat, is indicative of several basic body function conditions. Therefore, the development of simple methods able to detect these alkaline ions is of outmost importance. In this study, we explored the electrochemical and optical properties of graphene quantum dots (GQDs) combined with the selective chelating ability of the crown ethers 15 -crown-5 and 18-crown-6, with the final aim to propose novel composites for the effective detection of these ions. The results obtained comparing the performances of the single GQDs and crown ethers with those of the GQDs-15crown-5 and GQDs-18-crown-6 composites, have demonstrated the superior properties of these latter. Electrochemical investigation showed that the GQDs based composites can be exploited for the potentiometric detection of $\mathrm{Na}^{+}$and $\mathrm{K}^{+}$ions, but selectivity still remains a concern. The nanocomposites showed the characteristic fluorescence emissions of GQDs and crown ethers. The GQDs-18-crown-6 composite exhibited ratiometric fluorescence emission behavior with the variation of $\mathrm{K}^{+}$concentration, demonstrating its promising properties for the development of a selective fluorescent method for potassium determination.
\end{abstract}

Keywords: graphene quantum dots; $\mathrm{K}^{+}$detection; $\mathrm{Na}^{+}$detection; fluorescent sensors; electrochemical sensors

\section{Introduction}

Sensors for the selective quantification of sodium and potassium ions are of crucial importance in clinical diagnostics [1-3]. These ions are essential electrolytes for human homeostasis and changes in their concentrations can be related to serious complications for health, such as compromised heart function [4], cystic fibrosis [5], kidney failure [6], diabetic ketoacidosis [7] and adrenal disorders [8]. Sodium is the most abundant cation present in extracellular fluids, with a concentration rate between 135 and $145 \mathrm{mEq} / \mathrm{L}$, while potassium represents the most abundant cation inside cells, with concentrations in the range of 140-150 mEq/L [9]. These two electrolytes have been described as the body's dynamic duo, since they work in concert for energy production and to maintain the body's fluid balance [10]. Molecular sodium-potassium pumps pull potassium into cells and push sodium out, thus creating a chemical battery that is able to drive nerve signal transmission and to activate muscle contraction. The evaluation of the concentrations of these electrolytes, not only in serum, but also in sweat and in dermis, can provide important information on the human body's hydration status [11,12]. Their quantification can provide important information for therapeutic interventions in elderly people, especially in clinical or nursing home settings, and for sports performance optimization. Thus, their precise, sensitive and, if possible, contemporaneous monitoring, could allow the early diagnosis 
of various physiological and pathological events and to track the efficacy of medical treatments for correct therapeutic interventions.

Even if the existing techniques allow the sensitive quantification of these electrolytes in blood and urine samples, their use is limited by their cost, speed and the large amount of sample required $[9,13]$. Moreover, the different existing analytical methods for their detection do not allow for continuous monitoring in remote and in resource-limited environments. Thus, easy, sensitive, rapid, and low-cost methods for the real-time tracking of $\mathrm{Na}^{+}$and $\mathrm{K}^{+}$concentration in biological fluids are required to ensure the diagnosis and medical treatment of this fast-moving disease. Several studies on the sensitive and rapid detection of these electrolytes in the biological fluids have been proposed, including the use of wearable electrochemical sensors based on conductive polymers [12,14], extended gate field effect transistor for multi-ions sensing [15], ion-selective optical sensors [16,17] and paper-based biosensors [18].

However, the achievement of a selective response in $\mathrm{Na}^{+}$or $\mathrm{K}^{+}$detection is still a challenge. One of the reasons for the difficulty of distinguishing the signals of the two ions lies in their similar chemical properties. The possibility to selectively detect these two ions can be achieved by taking advantage of their different Pauling ion radii $(1.02 \AA$ for sodium and $1.38 \AA$ for potassium) [19]. Among the different supra-molecular host molecules able to form stable host-guest complexes with ions in function of their size, crown ethers represent the standard due to their stronger affinities for alkali metal cations in solution [20]. Their cation selectivity is dependent on the size and charge density of the ion and the crown ether cavity and is mainly achieved by ion-dipole interactions [21]. Among them, 15-crown-5 and 18-crown-6 are good hosts for sodium and potassium ions, respectively, and have been investigated for the development of selective fluorescent, potentiometric and electrochemical sensors for the detection of these biologically important electrolytes in solution [22-26].

Graphene based materials, due to their high electrical conductivity and large planar area, exhibit favorable properties for biosensing and bioimaging applications [27-30]. Moreover, recent theoretical studies suggest that the existing crown ether configurations in graphene could afford a much larger binding energy [30]. A graphene-based fluorescence resonance energy transfer (FRET) sensor linked to an ion-selective crown ether revealed a detection limit of $\mathrm{K}^{+}$ion of about $1 \times 10^{-5} \mathrm{M}$ [23]. Reduced graphene oxide (rGO) covalently conjugated with 18 -crown- 6 ether displayed the ability to detect $\mathrm{K}^{+}$ions down to the micromolar range [25], while an electrochemical sensor based on 1-aza-18-crown-6 functionalized GO reported a detection limit for $\mathrm{K}^{+}$ion of $10^{-15} \mathrm{M}$ [26]. The next generation of the graphene family, the zero dimensional graphene quantum dots (GQDs), received significant interest within academia and industry over the last few years due to their remarkable physicochemical properties [31,32]. Unlike two-dimensional graphene, GQDs have a band gap because of the quantum size effect and exhibit stable and size-dependent photoluminescence, which allows for their application in photovoltaics, electro/photo/chemical catalysis, the fabrication of flexible devices and in biosensing [33-36].

In this study, we report, for the first time, the combination of the electrical and optical properties of GQDs and the selective chelating ability of the crown ethers 15-crown-5 and 18-crown-6 with the aim of developing sensitive electrochemical and fluorescent sensors for sodium and potassium ions. The covalent conjugation of GQDs with the selected crown ethers allows for the development of nanocomposites able to discriminate between the two ions because of their different binding mechanisms. The results of this study show that the electrochemical properties and the fluorescence behavior of these composite nanomaterials make possible and promising the development of simple, rapid, and effective tests for the detection of $\mathrm{Na}^{+}$and $\mathrm{K}^{+}$ions, with the fluorescent method also able to achieve a selective detection towards potassium ions. 


\section{Materials and Methods}

\subsection{Materials}

The chemical reagents and solvents employed in this study were purchased from commercial suppliers and used without any further purification. GQDs were obtained from pristine multi-walled carbon nanotubes (MWCNTs), following a previously reported procedure [37]. Briefly, MWCNTs were treated with a mixture of $\mathrm{HNO}_{3} / \mathrm{H}_{2} \mathrm{SO}_{4}$ (1:3 ratio) in an ultrasonic bath at $60^{\circ} \mathrm{C}$ for four days. The suspension was diluted with deionized water and filtered on a $0.1 \mu \mathrm{m}$ Millipore membrane. The filtrate was treated with a solution of $\mathrm{NaOH}$ until it reached a neutral $\mathrm{pH}$ and was purified using dialysis bags (12 KDa). The number of acidic groups, as evaluated by titration analysis, was found to be of $2.37 \mathrm{mmol} / \mathrm{g}$.

\subsection{Synthesis of GQDs-15-Crown-5 and GQDs-18-Crown-6}

The composite nanomaterials were synthesized as follows. A water solution of GQDs (30 mg/30 mL) was added to $10 \mathrm{~mL}$ of dimethylformamide (DMF) and, after removal of water under reduced pressure, the mixture was treated with $\mathrm{N}$-(3-dimethylaminopropyl)N-ethylcarbodiimide hydrochloride (EDC $\cdot \mathrm{HCl}, 0.14 \mathrm{mmol})$, triethylamine (ETA, $0.14 \mathrm{mmol})$ and left to stir at room temperature for $15 \mathrm{~min}$. Hydroxyl benzotriazole (HOBt, $0.14 \mathrm{mmol}$ ) and a catalytic amount of 4-dimethylaminopyridine (DMAP) were then added and the mixture was left to stir for an additional $1 \mathrm{~h}$. The suspension was then treated with of 2-aminomethyl-15-crown-5 (0.12 mmol) for GQDs-15-crown-5 synthesis or 2-aminomethyl 18-crown-6 (0.1 mmol) for GQDs-18-crown-6 synthesis and left to stir at room temperature for four days. The obtained suspensions were then diluted with deionized water and purified using dialysis bags with MW of 12KDa until no organic material was present in the washing solutions. The degree of functionalization was evaluated on a known amount of sample (dried under vacuum at $60^{\circ} \mathrm{C}$ ) by thermogravimetric analysis, under argon atmosphere.

\subsection{Microstructural, Optical and Electrochemical Characterizations}

Thermogravimetric studies were carried out at $10{ }^{\circ} \mathrm{C} / \mathrm{min}$, from 100 to $1000{ }^{\circ} \mathrm{C}$, in argon atmosphere using TGA Q500 (TA instruments). The size determination was performed by dynamic light-scattering (DLS) analysis using the Zetasizer 3000 instrument (Malvern), equipped with a $632 \mathrm{~nm}$ HeNe laser and operating at a 173-degree detector angle. Infrared spectra were registered using a Perkin Elmer Spectrum 100 spectrometer, equipped with a universal ATR sampling accessory; spectra were recorded without any preliminary treatment, at room temperature, from 4000 to $600 \mathrm{~cm}^{-1}$, with a resolution of $4.0 \mathrm{~cm}^{-1}$. Micro-Raman spectra were measured in VV backscattering geometry using a LabRam HR 800 spectrometer. The spectra were recorded with an excitation wavelength of a $532 \mathrm{~nm}$ semiconductor diode laser, a $50 \times$ objective and a $77 \mathrm{~K}$ cooled charged couple device detector.

Photoluminescence (PL) measurements were performed at room temperature using a spectrofluorometer NanoLog modular (Horiba), under excitation with a xenon lamp; the GQD based nanomaterials were analyzed at the concentration of $100 \mathrm{ng} / \mathrm{mL}$. To test the electrochemical properties, screen-printed commercial electrodes (SPCE) were functionalized directly by drop casting 18 microliters of a dispersion $(1 \mathrm{mg} / \mathrm{mL})$ of the GQDs based nanomaterials on the working electrode of SPE, and then left to dry overnight at room temperature. Electrochemical experiments were performed using a DropSens $\mu$ Stat 400 potentiostat. The electrochemical behavior of the developed sensors was investigated in a deionized water solution at $\mathrm{pH} 7$, in the presence and absence of $\mathrm{KCl}$ or $\mathrm{NaCl}$, performing potentiometric measurements, i.e., measuring the potential difference between the two electrodes under the conditions of no current flow. 


\section{Results and Discussion}

\subsection{Synthesis and Characterization of GQDs-15-Crown-5 and GQDs-18-Crown-6}

The synthesis and characterization of the initial GQDs, obtained by acidic oxidation and chemical exfoliation of MWCNTs, was previously reported [37]. These carbon nanodots, with a weighted size distribution of $4.8 \mathrm{~nm}$, are characterized by the presence of many oxygen containing functional groups, due to the strong acidic treatment during their synthesis. The presence of carboxyl functionalities allows the covalent conjugation of GQDs with crown ethers functionalized with nucleophilic amino groups.

The coupling reaction between 2-aminomethyl-15-crown-5 $\left(\mathrm{NH}_{2}-15\right.$-crown-5) or 2aminomethyl-18-crown-6 ( $\mathrm{NH}_{2}-18$-crown-6) to the surface of GQDs was performed by activating the carboxylic functionalities present on the graphene surface using EDC/HOBt and 0.1 equivalent of DMAP, thus leading to the formation of amide bonds between these groups and the crown ethers (Scheme 1).

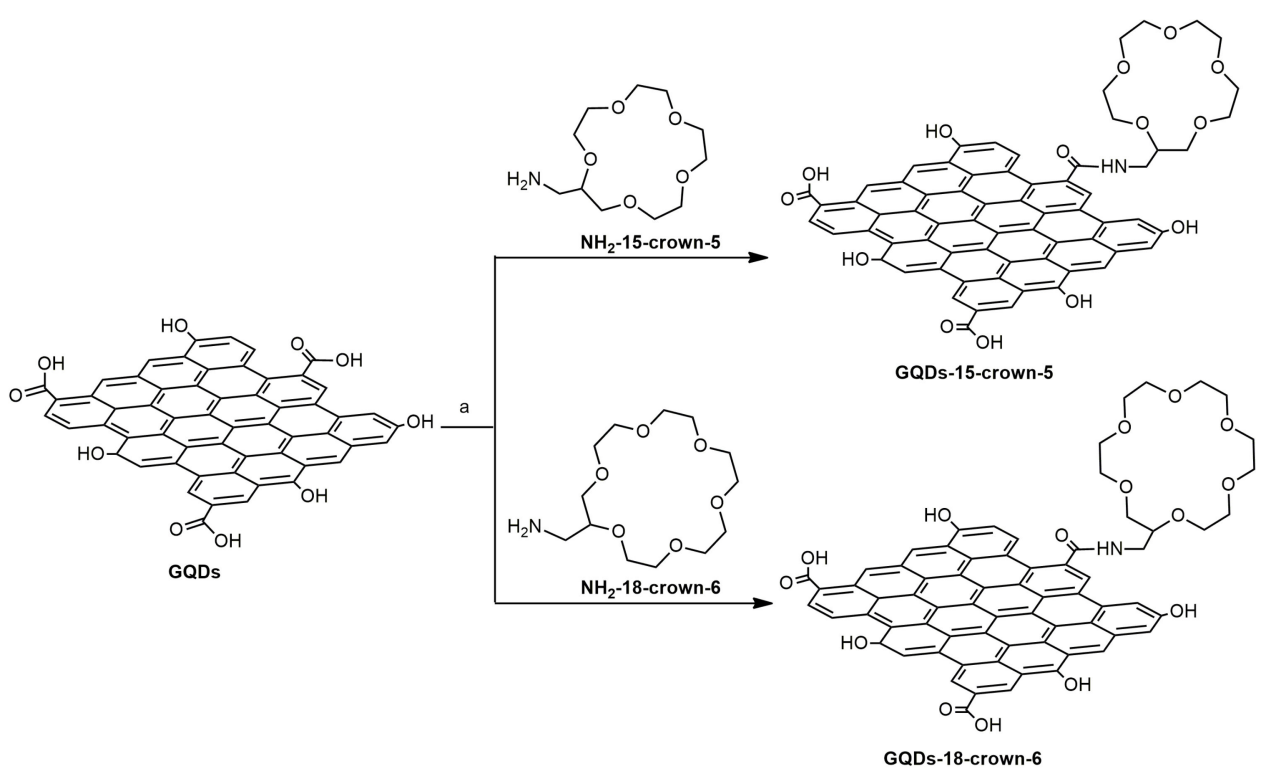

Scheme 1. Synthesis of GQDs-15-crown-5 and GQDs-18-crown-6. Reagents and conditions: (a) 2-aminomethyl-crown ether, EDC.HCl, HOBt, DMAP, DMF, r. t., 4 d.

After the removal of unreacted reagents and reaction solvent by dialysis, the effectiveness of the coupling reactions leading to GQDs-15-crown-5 and GQDs-18-crown-6, as well as the amount of crown ethers moieties loaded onto GQDs, was investigated by Fourier transform infrared spectroscopy (FTIR) and by thermogravimetric analysis (TGA) (Figure 1). These characterization techniques are widely used to prove the effectiveness of organic functionalization for carbon based nanomaterials. In particular, FTIR spectroscopy allows for proving the covalent functionalization of organic molecules to the surface of graphene based materials. The FTIR spectrum of GQDs (Figure 1a) shows the presence of a peak at $1610 \mathrm{~cm}^{-1}$ due to the stretching of the $\mathrm{C}=\mathrm{O}$ group of the carboxyl functionality and a large band at $3450 \mathrm{~cm}^{-1}$ related to the stretching of the $\mathrm{O}-\mathrm{H}$ bond. These peaks are indicative of the presence of many oxygen-containing groups on the surface of GQDs. The spectra of the conjugated samples GQDs-15-crown-5 and GQDs-18-crown-6 show the additional representative peaks at $1120 \mathrm{~cm}^{-1}$ which can be assigned to the ether functional group (C-O-C) of crown ethers, while the additional peaks at $1640 \mathrm{~cm}^{-1}$ are attributable to the newly formed amide bonds between the crown ethers and the GQDs. 


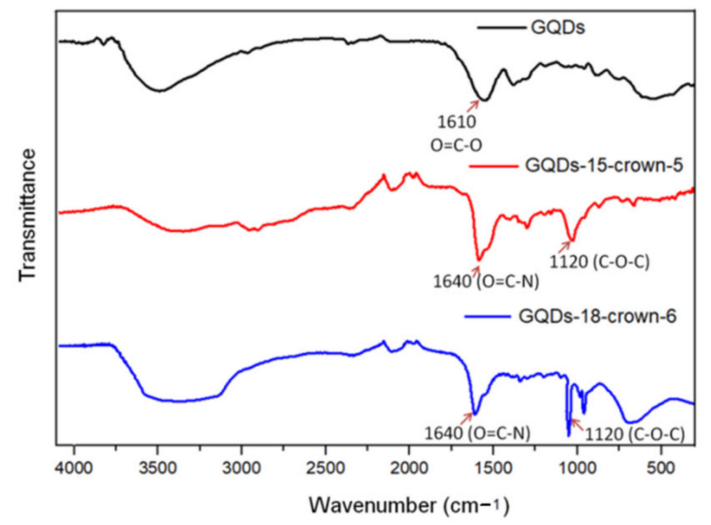

(a)

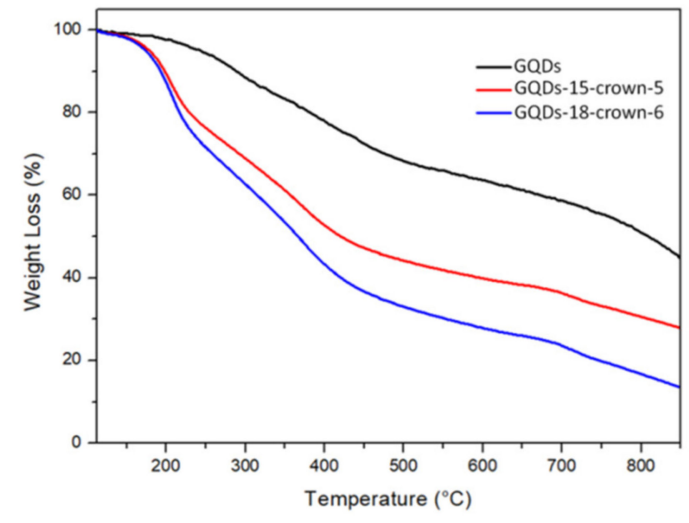

(b)

Figure 1. (a) FTIR spectra of the starting GQDs, GQDs-15-crown-5 and GQDs-18-crown-6; (b) TGA curves of GQDs, GQDs-15-crown-5 and GQDs-18-crown-6. All experiments were performed under Ar atmosphere.

The TGA curves of the GQDs and of the corresponding crown ethers functionalized GQDs, performed under inert atmosphere, show an increase inweight loss for both conjugated samples, the amounts of which, as calculated at $500{ }^{\circ} \mathrm{C}$, were found to be of $24 \mathrm{wt} \%$ and $35.1 \mathrm{wt} \%$ for GQDs-15-crown-5 and GQDs-18-crown-6, respectively (Figure 1b). Moreover, the different profiles of the TGA curves of the conjugated systems, confirm that the deep chemical modifications occurred on the nanomaterials after the functionalization processes.

Raman spectroscopy is a very useful technique forinvestigating the level of disorder in graphene based materials. Raman spectra of GQDs and of the crown ethers conjugated samples confirmed that the structural modification occurred on the conjugated samples after the functionalization procedures (Figure 2a). In particular, we have investigated the variations in the intensities of the D-band (ca. $1360 \mathrm{~cm}^{-1}$ ) and G-band (ca. $1590 \mathrm{~cm}^{-1}$ ) of the starting GQDs, GQDs-15-crown-5 and GQDs-18-crown-6 after exciting the samples at $532 \mathrm{~nm}$. The D-band is related to local defects that originate from structural imperfections of the aromatic networks, while the G-band is considered as the Raman fingerprint of the graphitic crystalline arrangements [38]. The $\mathrm{I}_{\mathrm{D}} / \mathrm{I}_{\mathrm{G}}$ ratio is usually used to evaluate the presence of disorder in $\mathrm{sp}^{2}$ hybridized carbon systems and to prove the effectiveness of the functionalization reactions. This value, which is equal to 1.18 for the starting GQDs, decreased for both functionalized samples to 1.068 and 1.065 for GQDs-15-crown-5 and GQDs-18-crown-6, respectively. These values demonstrated that the functionalization procedures enhanced the crystalline structure of the $\mathrm{sp}^{2}$ hybridized carbon network.

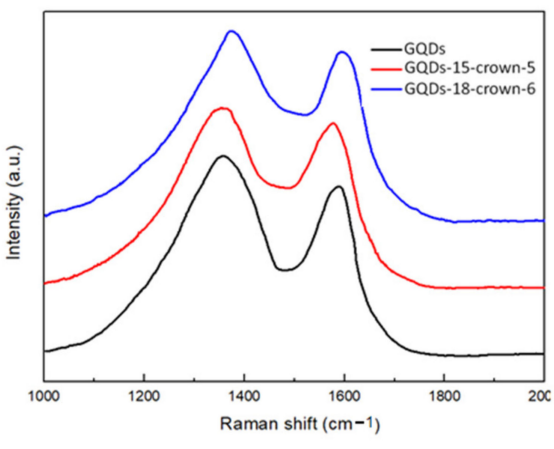

(a)

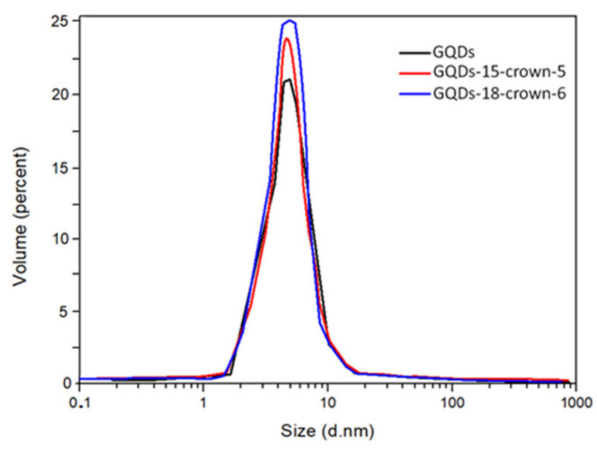

(b)

Figure 2. (a) Raman spectra of GQDs, GQDs-15-crown-5 and GQDs-18-crown-6; (b) DLS measurements of GQDs, GQDs-15-crown-5 and GQDs-18-crown-6; all experiments were performed in deionized water. 
To verify if the particle size of the GQDs have been maintained after the functionalization processes, we have investigated the conjugated samples by DLS (Figure $2 b$ ). This technique allows for evaluating the size distribution profile of small particles by measuring the random changes in the intensity of light scattered from the particles' suspension. The volume-weighted DLS measurements, performed on dispersions of the conjugated samples in deionized water, showed single size populations centered at 4.93 and $4.88 \mathrm{~nm}$, for GQDs15-crown-5 and GQDs-18-crown-6, respectively. The observed values are superimposable with that of the starting GQDs, thus confirming that no particles aggregation occurred on the nanomaterials after the functionalization procedure.

\subsection{Electrochemical Studies}

The electrochemical characteristics of the nanocomposite samples have been investigated to evaluate their possible use as ionophores in membrane-free screen-printed potentiometric sensors. Crown ethers have been previously reported as ionophores in ion-selective electrodes (ISEs) [39]. Potentiometric tests have been performed in the $\mathrm{Na}^{+}$ and $\mathrm{K}^{+}$concentrations range of 1 to $1000 \mathrm{mM}$ in deionized water at $\mathrm{pH} 7$. Figure 3 reports the behavior of GQDs-18-crown-6/SPCE towards $\mathrm{K}^{+}$ions, by immersing the electrode in potassium ion solutions of different concentrations.

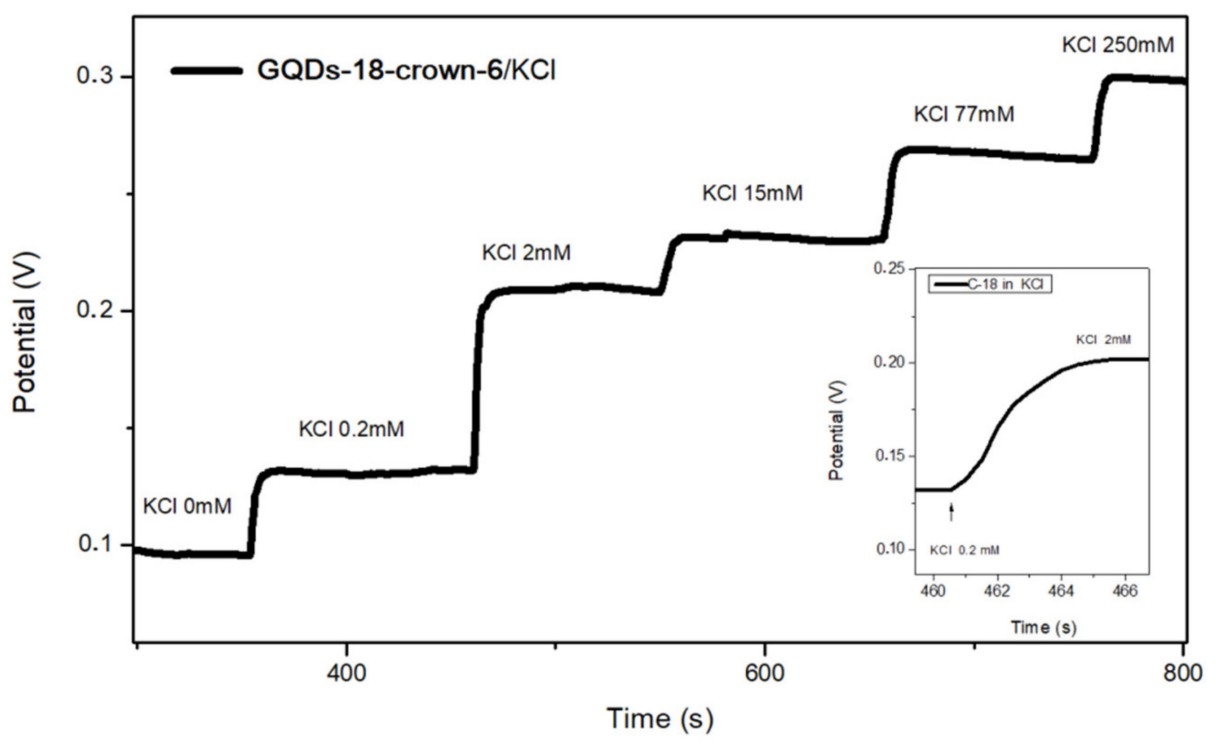

Figure 3. Potentiometric tests of GQDs-18-crown-6/SPCE towards $\mathrm{K}^{+}$ions with different concentrations. In the inset is shown an enlargement of the step leading from 0.2 to $2 \mathrm{mM}$ in the metal ions concentration.

The electrode response time was estimated by recording the time needed to attain a steady state potential after a sudden tenfold increase in the metal ions concentration [40]. Composite based electrodes showed a fast response (less than 4s, see inset in Figure 3). The good sensing properties described may be attributed to the synergistic effect between the high electron transfer properties of GQDs and the chelating ability of crown ethers within the electrode matrix. This behavior was observed also using the GQDs-15-crown-5/SPCE sensor.

Figure 4 reports the calibration curve of GQDs-18-crown-6/SPCE for the detection of $\mathrm{K}^{+}$ions. The comparison with the single GQDs and the 18-crown- 6 based sensor confirms that the potentiometric response is largely enhanced by the presence of the GQDs-18crown- 6 composite as ionophore. Moreover, a linear response with the increase of the $\mathrm{K}^{+}$concentration was recorded. The nanocomposite-based sensors showed a super-high Nernstian slope value $(75.3 \mathrm{mV} /$ decade). This anomalous high response has been already reported for chelating ionophores, such as crown ethers [41]. However, the data reported in the same plot in the presence of $\mathrm{Na}^{+}$evidenced clearly that is not possible to discriminate 
between the two ions. The same findings, in terms of response and selectivity, were found for the GQDs-15-crown-5/SPCE sensor.

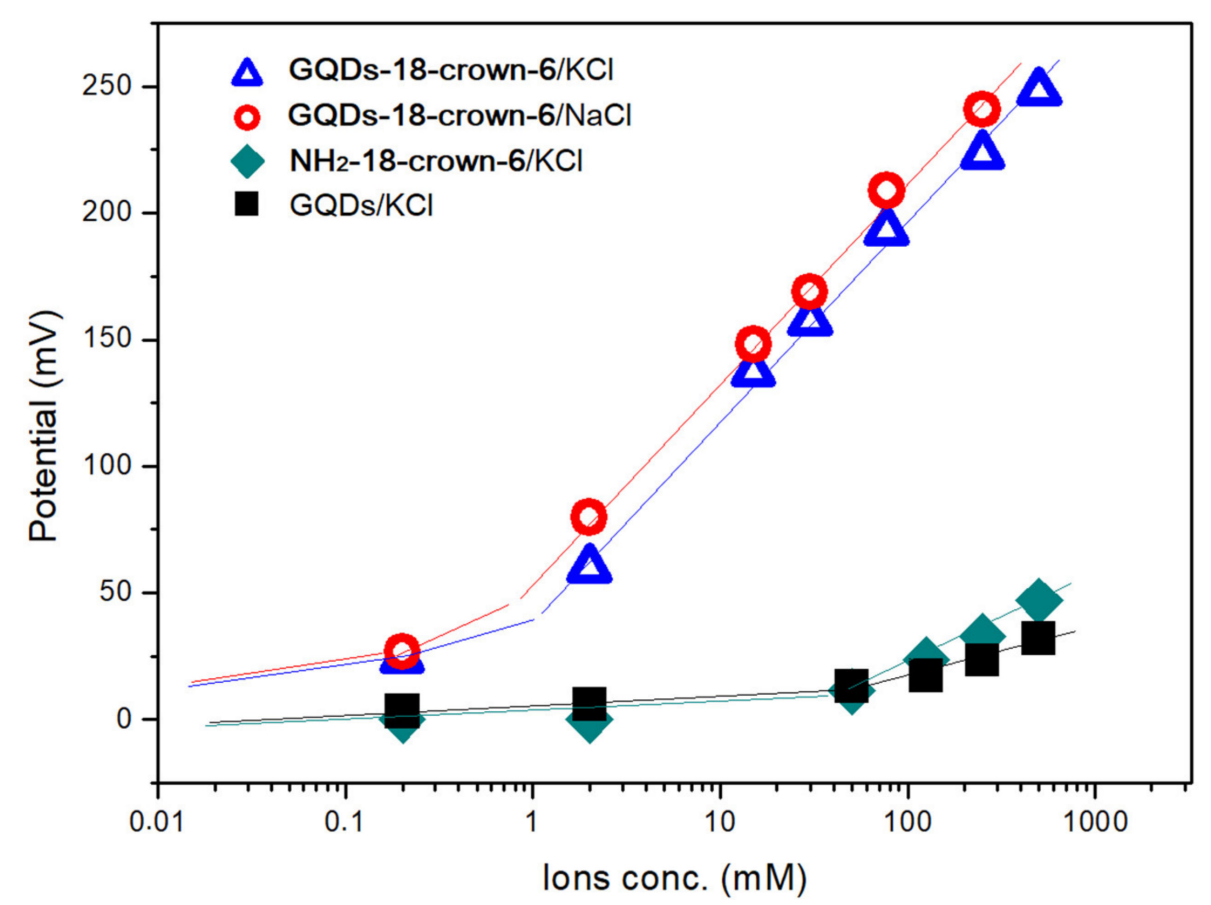

Figure 4. Comparison of the electrochemical response for GQDs-18-crown-6/SPCE, towards $\mathrm{K}^{+}$and $\mathrm{Na}^{+}$ions.

EIS measurements (Figure S1) provided further information helpful to understand the electrochemical sensing mechanism. The strong decrease in the charge transfer resistance for GQDs/SPCE reveals the more conductive nature and faster electron transfer rate as compared to the bare SPCE. These data demonstrated that the GQDs-18-crown-6/SPCE sensor benefits of the presence of GQDs in the composite formulation. Indeed, despite the low conductivity of 18-crown-6 moiety, the GQDs-18-crown-6/SPCE exhibits a good charge transfer rate, which is an important parameter forenhancing the performance of the electrochemical sensor. Combining EIS data with the potentiometric results, it appears that the complexation of $\mathrm{Na}^{+}$and $\mathrm{K}^{+}$ions occurring at the electrode-solution interface, facilitating the fast transfer into the electrode surface and the fast electron transfer, are at the origin of the good response of the GQDs-18-crown-6/SPCE to the changes of concentration of these ions in solution. To evaluate the stability of the GQDs-18-crown-6 based electrode, we performed repetitive measurements. We found that, for tests carried in successive days, the response of the electrode was nearly unchanged (Figure S2).

\subsection{Photoluminescence Studies}

The photoluminescence properties of the synthesized nanomaterials were investigated by comparing the PL properties of the starting nanomaterials with those of the corresponding crown ethers conjugated samples in deionized water at the excitation wavelength of $360 \mathrm{~nm}$ (Figure 5). 


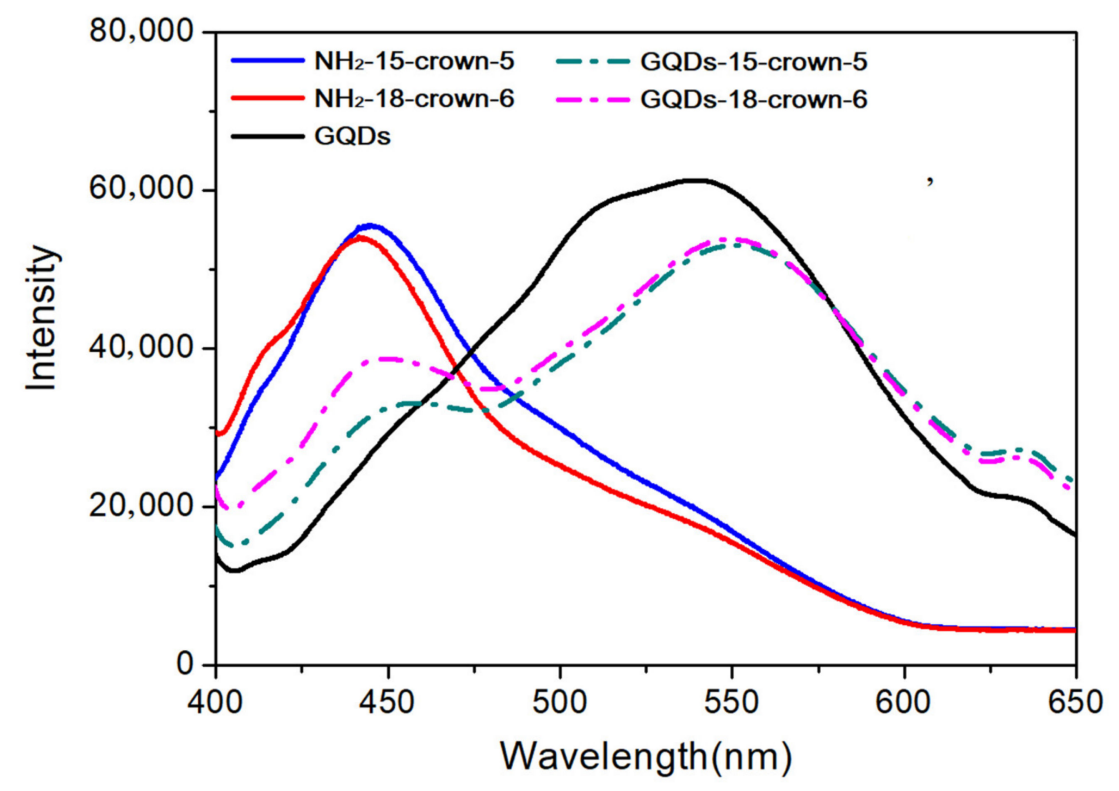

Figure 5. PL spectra of $\mathrm{NH}_{2}$-15-crown-5, $\mathrm{NH}_{2}$-18-crown-6, GQDs, GQDs-15-crown-5 and GQDs-18crown- 6 , at the $\lambda_{\text {exc }}$ of $360 \mathrm{~nm}$. All samples were tested at a concentration of $100 \mathrm{ng} / \mathrm{mL}$.

The GQDs-15-crown-5and GQDs-18-crown-6 samples show a strong emission peak with a maximum at $550 \mathrm{~nm}$ and a shoulder at around $450 \mathrm{~nm}$. This additional emission peak at the lower wavelength can be reasonably attributed to the crown ether components (see PL of $\mathrm{NH}_{2}-15$-crown-5 and $\mathrm{NH}_{2}-18$-crown-6 samples in Figure $\mathrm{S} 3$ ), in agreement with results reported in the literature for similar crown ether conjugates [22,42]. The observed blue-shift of emissions of the conjugated samples with respect to the starting GQDs can be attributed to the covalent conjugation with the crown ethers [43].

The fluorescence behavior in the presence of different concentrations of $\mathrm{Na}^{+}$and $\mathrm{K}^{+}$ ions was then investigated. Analogously to potentiometric studies, concentrations of the analytes in the range of 1 to $1000 \mathrm{mM}$ in deionized water were used. The photoluminescence performances of single GQDs and the free crown ethers (Figure S3) were firstly evaluated. As shown in Figure S3a,b, a slight increase in fluorescence was observed when increasing concentrations of the two ions were added to the GQDs solutions, regardless of the added salt. For the unbound crown ethers, the observed increase in emission (Figure S3c-f) is particularly evident for the complexation of the ion of interest with the corresponding crown ether $\left(\mathrm{K}^{+}\right.$for $\mathrm{NH}_{2}-18$-crown-6 and $\mathrm{Na}^{+}$for $\mathrm{NH}_{2}-15$-crown-5), as also reported in the literature for a similar system $[44,45]$.

The photoluminescence performances of the conjugated samples GQDs-15-crown-5 and GQDs-18-crown-6 are shown in Figure 6. The GQDs-15-crown-5 sample shows a clear affinity to $\mathrm{Na}^{+}$ions, affording enhancements of fluorescence intensity when increasing concentrations of this analyte were added to the system (Figure 6a); conversely, increasing additions of $\mathrm{K}^{+}$ion to the same system did not exert consistent changes in the fluorescence emission (Figure 6b). For the GQDs-18-crown-6 sample, a different PL behavior was observed (Figure $6 c, d$ ). Enhancements of fluorescence were recorded at $450 \mathrm{~nm}$ after the addition of increasing concentrations of $\mathrm{Na}^{+}$ion, while the addition of increasing concentrations of $\mathrm{K}^{+}$to the same system afforded anincreasing quenching of fluorescence at $550 \mathrm{~nm}$ and increasing in PL emission at $450 \mathrm{~nm}$. 


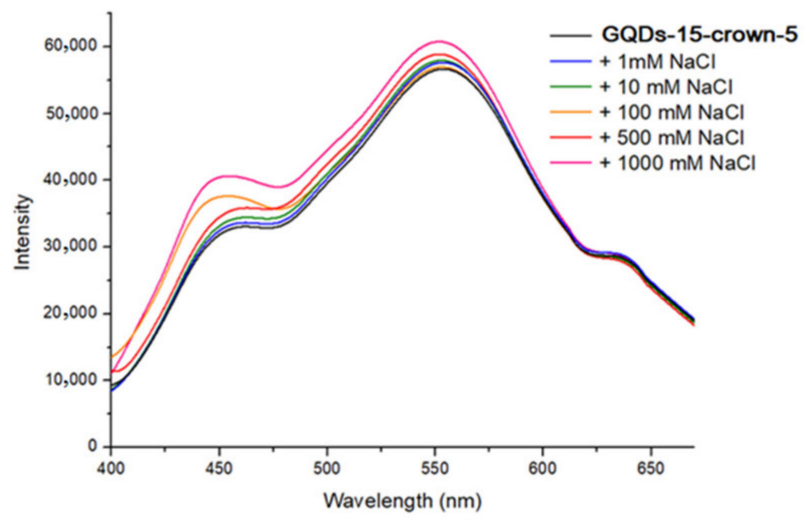

(a)

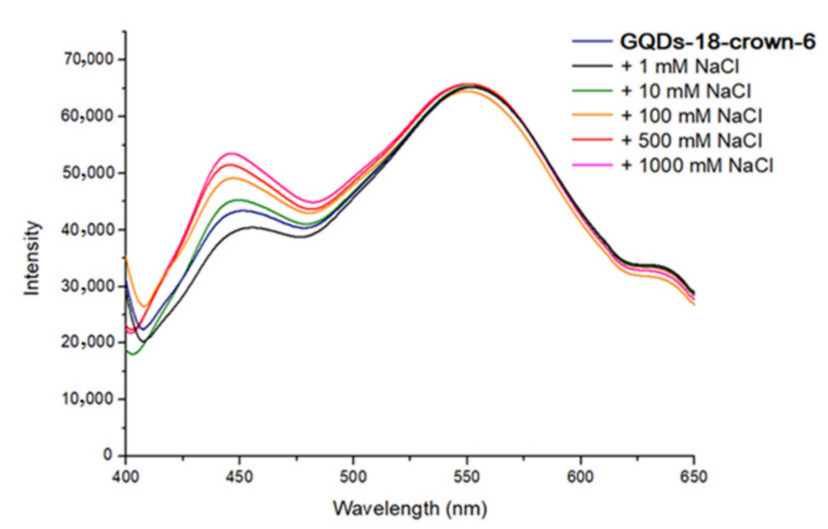

(c)

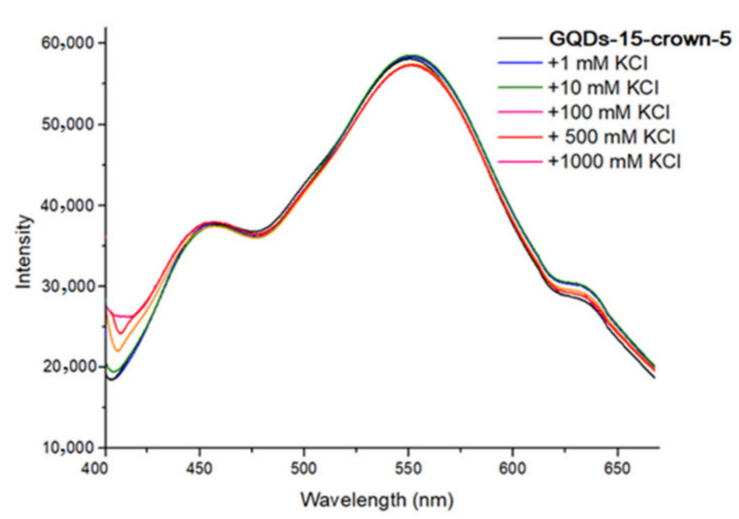

(b)

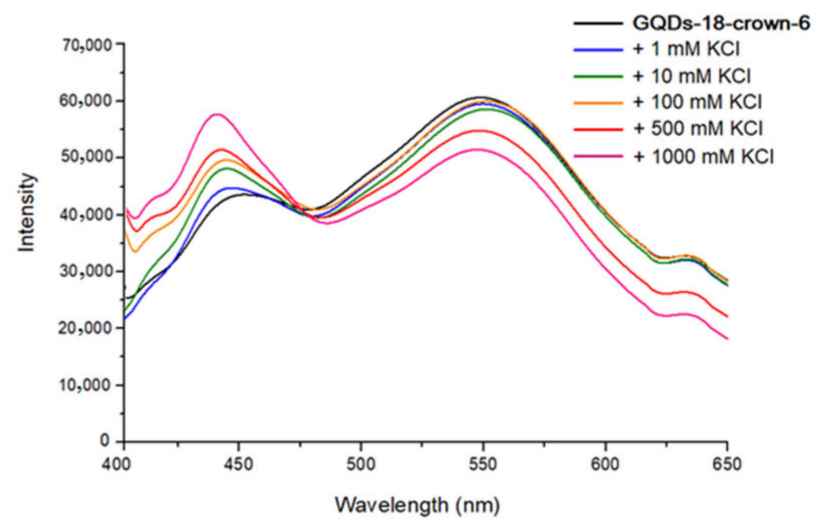

(d)

Figure 6. PL spectra of GQDs-15-crown-5 in the presence of different concentrations of (a) $\mathrm{NaCl}$ or (b) $\mathrm{KCl}$ and $\mathrm{PL}$ spectra of GQDs-18-crown-6 in the presence of different concentrations of (c) $\mathrm{NaCl}$ and (d) $\mathrm{KCl}$. All spectra were performed at the $\lambda_{\text {exc }}$ of $360 \mathrm{~nm}$ at the concentration of $100 \mathrm{ng} / \mathrm{mL}$.

Analogous photoluminescence behavior was observed for this sample, after addition of the same concentration (100 mM) of both ions (Figure S4). An increase in fluorescence was still recorded at $450 \mathrm{~nm}$, together with a quenching of fluorescence at $550 \mathrm{~nm}$, thus further confirming the PL mechanism of this sample in the presence of the $\mathrm{K}^{+}$ion.

The increase in PL emissions at $450 \mathrm{~nm}$ and the contemporary quenching of fluorescence at $550 \mathrm{~nm}$ allows for proposing the composite GQDs-18-crown-6 for a ratiometric sensing method for potassium ions detection. Ratiometric sensing, based on the ratio of fluorescence intensities at two wavelengths, results are more sensitive and reliable than those from a single fluorescence probe because it canprovide a built-in self-calibration as an internal standard [46]. Carbon dots-based nanomaterials have, in the past, been used to develop similar ratiometric systems [47]. From the results reported in Figure $6 c, d$, we computed the $\mathrm{I}_{450} / \mathrm{I}_{550}$ intensity ratio in the presence of various concentrations of the alkaline ions and reported these values vs. the ions concentrations (Figure 7). The increase of fluorescence intensity at $450 \mathrm{~nm}$ and the contemporary decrease at $550 \mathrm{~nm}$ results in the consequential increase of the $\mathrm{I}_{450} / \mathrm{I}_{550}$ intensity ratio with an increase in the $\mathrm{K}^{+}$concentration. Figure 7 also shows that the $\mathrm{I}_{450} / \mathrm{I}_{550}$ intensity ratio for $\mathrm{Na}^{+}$increases less than for $\mathrm{K}^{+}$in the same concentration range, thus suggesting that the GQDs-18-crown-6 composite displays a certain selectivity towards potassium ions. 


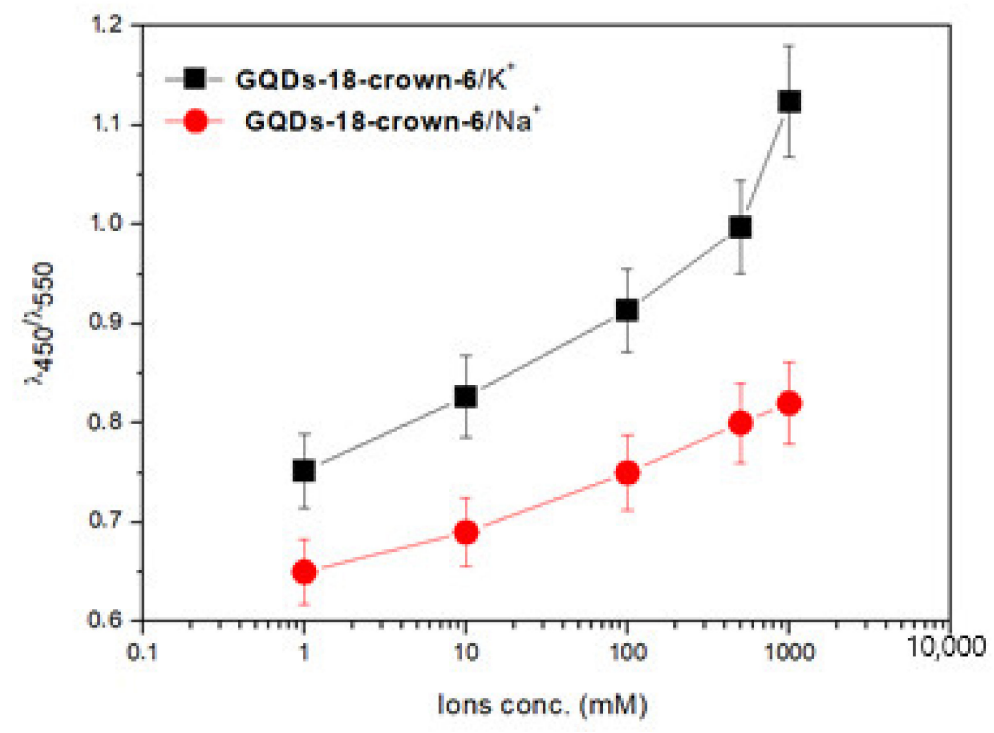

Figure 7. Ratio of fluorescence intensity $\left(\mathrm{I}_{450} / \mathrm{I}_{550}\right)$ for GQDs-18-crown-6 sample, measured as a function of the concentration of $\mathrm{K}^{+}$and $\mathrm{Na}^{+}$ions. Error bars indicate the standard deviation from experiments done in triplicate.

This behavior can be explained by considering a different complexation mechanism between the GQDs-18-crown- 6 composite and the $\mathrm{K}^{+}$ion. As also reported in the literature for similar systems [16], this ion can interact with the oxygens of the crown ether and with the amide oxygens nearby the GQDs surface; the interaction with the oxygen containing groups of the GQDs surface is also expected. This conformational change stabilizes the complex with the $\mathrm{K}^{+}$ion and increases the interaction of the crown ether with the GQDs surface. As also reported in other studies, the non-covalent interaction of small molecules with the GQDs surface can lead to the observed quenching phenomenon $[48,49]$. On the basis of the observed fluorescence behavior, we propose the fluorescence mechanism for the GQDs-18-crown-6 composite system in the presence of $\mathrm{Na}^{+}$or $\mathrm{K}^{+}$ions, as shown in Scheme 2 .

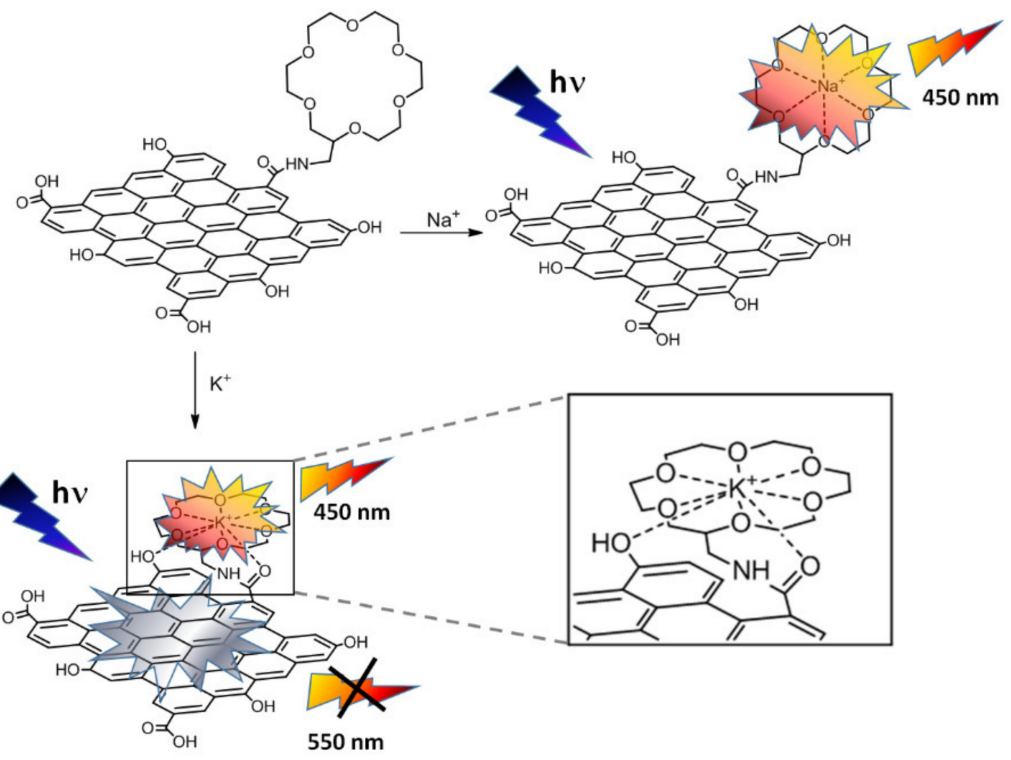

Scheme 2. Proposed mechanism of binding of $\mathrm{K}^{+}$and $\mathrm{Na}^{+}$ions to GQDs-18-crown- 6 composite. The different behavior to irradiation in the presence of two ions is well evident. 
As is well evident, binding of both $\mathrm{Na}^{+}$and $\mathrm{K}^{+}$ions turn on the fluorescent peak related to the GQDs-18-crown-6. However, in the presence of $\mathrm{K}^{+}$ions, quenching of the fluorescent peak related to GQDs moiety occurs too, thus enabling the ratiometric fluorescence sensing. This observation leads us to hypothesize that the interaction of $\mathrm{Na}^{+}$ with the oxygens of the crown ether and also with the amide oxygens nearby the GQDs surface, as supposed for $\mathrm{K}^{+}$, does not occur. This different behavior could include both steric and/or electronic factors. Regardless, it appears that GQDs serves as reference fluorophore, while the crown ether serves as a recognition unit with a turn-on response. We are extending this study to other ions to better understand the origin of the differences observed between the investigated alkaline ions.

\section{Conclusions}

Sensors for the selective quantification of sodium and potassium ions are of crucial importance in clinical diagnostics, since changes in the concentrations of these ions can be related to serious complications for human health. In this study, we have investigated the electrochemical and optical properties of GQDs and the selective chelating ability of the crown ethers 15-crown-5 and 18-crown-6, for the development of fluorescent and electrochemical sensors for the determination of these two ions.

The electrochemical performance of the synthesized nanocomposites, evaluated at the concentration range up to $1 \mathrm{mM}$, demonstrated high sensitivity; however, low selectivity between these ions was observed.

Both nanocomposites show the characteristic fluorescence emissions of GQDs and crown ethers under a single excitation wavelength. Interestingly, for the GQDs-18-crown-6 sample, a ratiometric fluorescence emission behavior with the variation of $\mathrm{K}^{+}$concentration was observed.

The observed fluorescence behavior of this sample can allow for the development of simple, rapid, and effective tests for the selective detection of potassium over sodium ion. The extension of this study to other ions, also by electrochemiluminescence (ECL) tests, will allow for a better understanding of the origin of the differences observed between the investigated ions.

Supplementary Materials: The following are available online at https://www.mdpi.com/article/10 .3390/nano11112897/s1, Figure S1: EIS measurements for GQDs-18-crown-6/SPCE, GQDs/SPCE, bare SPCE, Figure S2: Reliability of GQDs-18-crown- 6 basedelectrode in different measurements, Figure S3: PL spectra of GQDs, $\mathrm{NH}_{2}-15-$ crown- 5 and $\mathrm{NH}_{2}-18$-crown- 6 in the presence of different concentrations of $\mathrm{NaCl}$ or KCl, Figure S4. PL spectra of GQDs-18-crown-6 and ofthe same samplein the presence of $\mathrm{NaCl} 100 \mathrm{mM}, \mathrm{KCl} 100 \mathrm{mM}$ or in the presence of $\mathrm{KCl}$ and $\mathrm{NaCl}, 100 \mathrm{mM}$.

Author Contributions: Conceptualization, D.I. and C.E.; methodology, A.F. and C.C.; validation, D.I., C.B. and G.N.; formal analysis, C.C., C.B. and C.E.; investigation, D.I. and G.N.; resources, C.E. and G.N.; data curation, A.F. and C.C.; writing-original draft preparation, D.I. and C.E.; writing-review and editing, D.I. and C.E.; supervision, G.N. All authors have read and agreed to the published version of the manuscript.

Funding: This research received no external funding.

Institutional Review Board Statement: Not applicable.

Informed Consent Statement: Not applicable.

Data Availability Statement: The data presented in this study are available in the article.

Conflicts of Interest: The authors declare no conflict of interest.

\section{References}

1. Pohl, H.R.; Wheeler, J.S.; Murray, H.E. Sodium and Potassium in Health and Disease. In Interrelations between Essential Metal Ions and Human Diseases; Sigel, A., Sigel, H., Sigel, R., Eds.; Springer: Dordrecht, The Netherlands, 2013; Volume 13, pp. $29-47$.

2. Lim, H.R.; Kim, Y.-S.; Kwon, S.; Mahmood, M.; Kwon, Y.T.; Lee, Y.; Lee, S.M.; Yeo, W.-H. Wireless, Flexible, Ion-Selective Electrode System for Selective and Repeatable Detection of Sodium. Sensors 2020, 20, 3297. [CrossRef] [PubMed] 
3. Ghafar-Zadeh, E. Wireless Integrated Biosensors for Point-of- Care Diagnostic Applications. Sensors 2015, 15, 3236-3261. [CrossRef] [PubMed]

4. Ferreira, J.P.; Butler, J.; Rossignol, P.; Pitt, B.; Anker, S.D.; Kosiborod, M.; Lund, L.H.; Bakris, G.L.; Weir, M.R.; Zannad, F. Abnormalities of Potassium in Heart Failure: JACC State-of-the-Art Review. J. Am. Coll. Cardiol. 2020, 75, 2836-2850. [CrossRef] [PubMed]

5. Scurati-Manzoni, E.; Fossali, E.F.; Agostoni, C.; Riva, E.; Simonetti, G.D.; Zanolari-Calderari, M.; Bianchetti, M.G.; Lava, S.A.G. Electrolyte abnormalities in cystic fibrosis: Systematic review of the literature. Pediatr. Nephrol. 2014, 29, 1015-1023. [CrossRef]

6. Piccoli, G.B.; Capobianco, M.; Odetto, L.; Deagostini, M.C.; Consiglio, V.; Radeschi, G. Acute renal failure, severe sodium and potassium imbalance and sudden tetraplegia. NDT Plus 2010, 3, 247-252. [CrossRef]

7. Usman, A. Initial Potassium Replacement in Diabetic Ketoacidosis: The Unnoticed Area of Gap. Front. Endocrinol. 2018, 9, 109. [CrossRef]

8. Fraser, R. Disorders of the adrenal cortex: Their effects on electrolyte metabolism. Clin. Endocrinol. Metab. 1984, 13, 413-430. [CrossRef]

9. Garcia, R.A.; Vanelli, C.P.; dos Santos Pereira, O.; do Amaral Corrêa, J.O. Comparative analysis for strength serum sodium and potassium in three different methods: Flame photometry, ion-selective electrode (ISE) and colorimetric enzymatic. J. Clin. Lab. Anal. 2018, 32, 22594. [CrossRef]

10. Poulsen, H.; Nissen, P. The Inner Workings of a Dynamic Duo. Science 2012, 335, 416-417. [CrossRef]

11. Jiang, N.; Yetisena, A.K.; Linhart, N.; Flisikowski, K.; Dong, J.; Dong, X.; Butt, H.; Jakobi, M.; Schnieke, A.; Koch, A.W. Fluorescent dermal tattoo biosensors for electrolyte analysis. Sens. Actuators B Chem. 2020, 320, 128378. [CrossRef]

12. Pirovano, P.; Dorrian, M.; Shinde, A.; Donohoe, A.; Brady, A.J.; Moyna, N.M.; Wallace, G.; Diamond, D.; McCaula, M. A wearable sensor for the detection of sodium and potassium in human sweat during exercise. Talanta 2020, 219, 121145. [CrossRef]

13. Banerjee, P.; Prasad, B. Determination of concentration of total sodium and potassium in surface and ground water using a flame photometer. Appl. Water Sci. 2020, 10, 113. [CrossRef]

14. Stekolshchikova, A.A.; Radaev, A.V.; Orlova, O.Y.; Nikolaev, K.G.; Skorb, E.V. Thin and Flexible Ion Sensors Based on Polyelectrolyte Multilayers Assembled onto the Carbon Adhesive Tape. ACS Omega 2019, 4, 15421-15427. [CrossRef]

15. Kabaa, E.A.; Abdulateef, S.A.; Ahmed, N.M.; Hassan, Z.; Sabah, F.A.A. novel porous silicon multi-ions selective electrode based extended gate field effect transistor for sodium, potassium, calcium, and magnesium sensor. Appl. Phys. A 2019, $125,753$. [CrossRef]

16. Jarolımova, Z.; Vishe, M.; Lacour, J.; Bakker, E. Potassium ion-selective fluorescent and pH independent nanosensors based on functionalized polyether macrocycles. Chem. Sci. 2016, 7, 525. [CrossRef]

17. $\mathrm{Xu}, \mathrm{X} . ; \mathrm{Xu}, \mathrm{H}$.; Ji, H.-F. From New fluorescent probes for the detection of mixed sodium and potassium metal ions. Chem. Comm. 2001, 20, 2092-2093. [CrossRef]

18. Chard, A.N.; Trinies, V.; Edmonds, C.J.; Sogore, A.; Freeman, M.C. The impact of water consumption on hydration and cognition among schoolchildren: Methods and results from a crossover trial in rural Mali. PLoS ONE 2019, 14, 0210568. [CrossRef]

19. Takami, T.; Son, J.W.; Lee, J.K.; Park, B.H.; Kawai, T. Separate detection of sodium and potassium ions with sub-micropipette probe. Jpn. J. Appl. Phys. 2011, 50, 08LB13. [CrossRef]

20. Pedersen, C.J. Cyclic polyethers and their complexes with metal salts. J. Am. Chem. Soc. 1967, 89, 2495-2496. [CrossRef]

21. Zhou, D.; Hao, H.; Ma, Y.; Zhong, H.; Dai, Y.; Cai, K.; Mukherjee, S.; Liu, J.; Bian, H. Specific Host-Guest Interactions in the Crown Ether Complexes with $\mathrm{K}^{+}$and $\mathrm{NH}_{4}{ }^{+}$Revealed from the Vibrational Relaxation Dynamics of the Counteranion. J. Phys. Chem. B 2020, 124, 9154-9162. [CrossRef]

22. Zhou, X.; Su, F.; Tian, Y.; Youngbull, C.; Johnson, R.H.; Meldrum, D.R. A New Highly Selective Fluorescent K ${ }^{+}$Sensor. J. Am. Chem. Soc. 2011, 133, 18530-18533. [CrossRef]

23. Wei, W.; Xu, C.; Ren, J.; Xu, B.; Qu, X. Sensing metal ions with ion selectivity of a crown ether and fluorescence resonance energy transfer between carbon dots and graphene. Chem. Commun. 2012, 48, 1284-1286. [CrossRef]

24. Gao, Y.; Sun, S.L.; Xu, H.L.; Zhao, L.; Su, Z.M. N-Methylbenzoaza-18-crown-6-ether derivatives as efficient alkali metal cations sensors: The dipole moment and first hyperpolarizability. RSC Adv. 2014, 4, 24433-24438. [CrossRef]

25. Olsen, G.; Ulstrup, J.; Chi, Q. Crown-Ether Derived Graphene Hybrid Composite for Membrane- Free Potentiometric Sensing of Alkali Metal Ions. ACS Appl. Mater. Interfaces 2016, 8, 37-41. [CrossRef]

26. Zhang, H.X.; Huang, Z.X.; Zhao, P.Y.; Hou, Y.; Guo, J.J.; Wu, Y.C. Crown ether functionalized graphene oxide as ultrasensitive electrochemical sensor for detection of potassium ions. Mater. Res. Express 2019, 6, 125095. [CrossRef]

27. Du, D.; Yuqi, Y.; Lin, Y. Graphene-based Materials for Biosensing and Bioimaging. MRS Bull. 2012, 37, 1290-1296. [CrossRef]

28. Wu, L.; Wang, J.; Yin, M.; Ren, J.; Miyoshi, D.; Sugimoto, N.; Qu, X. Reduced Graphene Oxide Upconversion Nanoparticle Hybrid for Electrochemiluminescent Sensing of a Prognostic Indicator in Early- Stage Cancer. Small 2014, 10, 330-336. [CrossRef]

29. Vasu, K.S.; Sridevi, S.; Sampath, S.; Sood, A.K. Non-Enzymatic Electronic Detection of Glucose Using Aminophenylboronic Acid Functionalized Reduced Graphene Oxide. Sens. Actuators B 2015, 221, 1209-1214. [CrossRef]

30. Guo, J.; Lee, J.; Contescu, C.I.; Gallego, N.C.; Pantelides, S.T.; Pennycook, S.K.; Moyer, B.A.; Chisholm, M.F. Crown ethers in graphene. Nat. Commun. 2014, 5, 5389. [CrossRef]

31. Iannazzo, D.; Ziccarelli, I.; Pistone, A. Graphene quantum dots: Multifunctional nanoplatforms for anticancer therapy. J. Mater. Chem. B 2017, 5, 6471-6489. [CrossRef] 
32. Iannazzo, D.; Celesti, C.; Espro, C. Recent Advances on Graphene Quantum Dots as Multifunctional Nanoplatforms for Cancer Treatment. Biotechnol. J. 2021, 16, 1900422. [CrossRef] [PubMed]

33. Mansuriya, B.D.; Altintas, Z. Applications of Graphene Quantum Dots in Biomedical Sensors. Sensors 2020, 20, 1072. [CrossRef] [PubMed]

34. Das, P.; Ganguly, S.; Banerjee, S.; Das, N.C. Graphene based emergent nanolights: A short review on the synthesis, properties and application. Res. Chem. Intermed. 2019, 45, 3823-3853. [CrossRef]

35. Das, P.; Ganguly, S.; Mondal, S.; Ghorai, U.K.; Maity, P.P.; Choudhary, S.; Gangopadhyay, S.; Dhara, S.; Banerjee, S.; Das, N.C. Dual doped biocompatible multicolor luminescent carbon dots for bio labeling, UV-active marker and fluorescent polymer composite. Luminescence 2018, 33, 1136-1145. [CrossRef]

36. Huang, H.; Liao, L.; Xu, X.; Zou, M.; Liu, F.; Li, N. The electron-transfer based interaction between transition metal ions and photoluminescent graphene quantum dots (GQDs): A platform for metal ion sensing. Talanta 2013, 117, 152-157. [CrossRef]

37. Giofrè, S.V.; Celesti, C.; Patanè, S.; Triolo, C.; Gulino, A.; Spitaleri, L.; Scalese, S.; Scuderi, M.; Iannazzo, D. Eco-Friendly 1,3-Dipolar Cycloaddition Reactions on Graphene Quantum Dots in Natural Deep Eutectic Solvent. Nanomaterials 2020, 10, 2549. [CrossRef]

38. Rajender, G.; Giri, P.K. Formation mechanism of graphene quantum dots and their edge state conversion probed by photoluminescence and Raman spectroscopy. J. Mater. Chem. C 2016, 4, 10852-10865. [CrossRef]

39. Suzuki, K.; Sato, K.; Hisamoto, H.; Siswanta, D.; Hayaski, K.; Kasahara, N.; Watanabe, K.; Yamamoto, N.; Sasakura, H. Design and synthesis of sodium ion-selective ionophores based on 16-crown-5 derivatives for an ion-selective electrode. Anal. Chem. 1996, 68, 208-215. [CrossRef]

40. Buck, R.P.; Lindner, E. Recommendations for nomenclature of ion selective electrodes (IUPAC Recommendations 1994). Pure Appl. Chem. 1994, 66, 2527-2536. [CrossRef]

41. Miyake, M.; Chen, L.D.; Pozzi, G.; Bühlmann, P. Ion-Selective Electrodes with Unusual Response Functions: Simultaneous Formation of Ionophore-Primary Ion Complexes with Different Stoichiometries. Anal. Chem. 2012, 84, 1104-1111. [CrossRef]

42. Sarfo, D.K.; Sivanesan, A.; Izake, E.L.; Ayoko, G.A. Rapid detection of mercury contamination in water by surface enhanced Raman spectroscopy. RSC Adv. 2017, 7, 21567-21575. [CrossRef]

43. Huang, C.L.; Huang, C.C.; Mai, F.D.; Yen, C.L.; Tzing, S.H.; Hsieh, H.T.; Ling, Y.C.; Chang, J.Y. Application of paramagnetic graphene quantum dots as a platform for simultaneous dual-modality bioimaging and tumor-targeted drug delivery. J. Mater. Chem. B 2015, 3, 651-664. [CrossRef]

44. Hausmann, D.; Kuzmanoski, A.; Feldmann, C. $\mathrm{MnBr}_{2} / 18$-crown-6 coordination complexes showing high room temperature luminescence and quantum yield. Dalton Trans. 2016, 45, 6541-6547. [CrossRef]

45. Merzlyakova, E.; Wolf, S.; Lebedkin, S.; Bayarjargal, L.; Neumeier, L.; Bartenbach, D.; Holzer, C.; Klopper, W.; Winkler, B.; Kappes, M.; et al. 18-Crown-6 Coordinated Metal Halides with Bright Luminescence and Nonlinear Optical Effects. Am. Chem. Soc. 2021, 143, 798-804. [CrossRef]

46. Li, P.; Li, S.F.Y. Recent advances in fluorescence probes based on carbon dots for sensing and speciation of heavy metals. Nanophotonics 2020, 10, 877-908. [CrossRef]

47. Lu, S.; Wu, D.; Li, G.; Lv, Z.; Chen, Z.; Chen, L.; Chen, G.; Xia, L.; You, J.; Wu, Y. Carbon dots-based ratiometric nanosensor for highly sensitive and selective detection of mercury(ii) ions and glutathione. RSC Adv. 2016, 6, 103169-103177. [CrossRef]

48. Iannazzo, D.; Pistone, A.; Celesti, C.; Triolo, C.; Patanè, S.; Giofrè, S.; Romeo, R.; Ziccarelli, I.; Mancuso, R.; Gabriele, B.; et al. A Smart Nanovector for Cancer Targeted Drug Delivery Based on Graphene Quantum Dots. Nanomaterials 2019, 9, 282. [CrossRef]

49. Wang, M.; Li, Y.; Wang, L.; Su, X. A label-free fluorescence nanosensor for the determination of adrenaline based on graphene quantum dots. Anal. Methods 2017, 9, 4434-4438. [CrossRef] 